\title{
Calidad Percibida por Usuarios de Hospitales del Programa de Reestructuración de Redes de Servicios de Salud de Colombia
}

\author{
Quality as perceived by people using hospitals in the Colombian \\ health service network restructuring programme
}

\author{
Gustavo A. Cabrera-Arana, León D. Bello-Parías y Jaime L. Londoño-Pimienta \\ Facultad Nacional de Salud Pública "Héctor Abad Gómez" de la Universidad de Antioquia. Medellín, \\ Colombia..gcabrera@guajiros.udea.edu.co, teoriasymodelos@hotmail.com
}

Recibido 2 Noviembre 2007/Enviado para Modificación 13 Julio 2008/Aceptado 28 Agosto 2008

RESUMEN

Objetivo Establecer una línea base de la calidad percibida por usuarios de hospitales objeto de intervención del Programa de reestructuración, rediseño y modernización de redes prestadoras de servicios de salud de Colombia.

Método Con instrumento validado para medir Percepción de Calidad Según Usuarios de Servicios de Salud, el PECASUSS, en agosto de 2006 se entrevistaron cara a cara 4021 usuarios de una muestra de los hospitales del Programa.

Resultados Los entrevistados, con media de 38 años, $70 \%$ mujeres, $60 \%$ con afiliación al régimen subsidiado, $55 \%$ de estrato uno y $53 \%$ atendidos en consulta general, valoraron mayoritariamente como bueno o muy bueno el nivel de dieciséis aspectos perceptibles de la calidad de los servicios. En una escala de 0,0 (pésimo) a 5,0 (excelente), calificaron la calidad con una media de 3,98 (DE: 0,8).

Conclusiones La calidad percibida por usuarios de los hospitales del Programa fue buena en general. El trato humanizado recibido de los funcionarios asistenciales fue la sub-dimensión de la calidad de la atención mejor valorada por los informantes.

Palabras Clave: Percepción, calidad de atención en salud, hospitales (fuente: DeCS, BIREME).

\section{ABSTRACT}

Objective Establishing a quality base-line as perceived by hospital users, subject to intervention by the health-service restructuring, redesign and modernisation programme.

Method 4021 users were interviewed face-to-face in August 2006 from a sample of hospitals from the programme using an instrument already validated for measuring health service users' perception of quality (PECASUSS). 
Results The interviewees (38,7\% mean age, $70 \%$ women, $60 \%$ affiliated to the subsided system, $55 \%$ from stratum 1 and $53 \%$ attended in general consultation) scored mostly as good or very good 16 different aspects perceived of the quality of services. Mean user quality score was $3,98(0,8 \mathrm{SD})$ on a score ranging from 0,0 (bad) to 5,0 (excellent).

Conclusions Quality was generally rated as good as perceived by people using the hospital programme. The humanised treatment received from the medical staff was given the highest score regarding quality of attention received by the interviewees.

Key Words: Perception, quality assurance, health care, hospital (source: MeSH, $N L M)$.

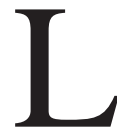

a República de Colombia suscribió en el año 2004 un contrato de cooperación con el Banco Interamericano de Desarrollo para ejecutar un Prodoras de servicios de salud del país. El Programa apoya la transformación de los hospitales, Instituciones Prestadoras de Servicios-IPS, de naturaleza pública, para mejorar su gestión y calidad (1). Como parte del seguimiento a la Política Nacional de Prestación de Servicios de Salud dicho Programa busca, además, implementar un sistema evaluativo de la calidad de los servicios prestados por estas IPS (2).

En este marco el Ministerio de la Protección Social de Colombia contrató el diseño e implementación de una metodología para evaluar el impacto del citado Programa en la calidad de los servicios según la percepción de los usuarios de las IPS intervenidas, comparando una línea base 2006 frente a dos mediciones, una intermedia de 2008 y una final de 2010 (3). Ante la inexistencia en el país de un instrumental confiable y válido para medir dicha percepción (4), se validó el instrumento denominado PECASUSS, acrónimo de Percepción de Calidad Según Usuarios de Servicios de Salud, en un proceso comunicado previamente (5). Este artículo reseña la metodología y los resultados de la medición de línea de base 2006.

\section{MÉTODOS}

El diseño de la metodología para efectuar las mediciones usó como referente el modelo de evaluación de programas e intervenciones de salud pública propuesto por Habicht, Victora y Vaughan (6). Análisis técnicos de la gestión y la calidad de las redes de prestadores de servicios, adelantados en el 2004 determinaron que las IPS elegibles para el Programa eran 301. Estas instituciones 
se distribuían en 25 departamentos del país; por niveles de complejidad de atención este marco tenía 220 (73 \%) hospitales del nivel I, 72 (24\%) del nivel II y 9 (3 \%) del nivel III.

Según muestra estimada con Epidat 3.0, con un error esperado menor a 5 $\%$ y teniendo en cuenta representatividad por nivel de complejidad, servicios ofertados y demanda registrada, las IPS finalmente seleccionadas para la línea de base fueron 48: 34 (71 \%) nivel I, 11 (23 \%) nivel II y 3 (6 \%) nivel III, localizadas en 17 de los 25 Departamentos del marco muestral (3). La Tabla 1 presenta la distribución y niveles de complejidad de la muestra.

Tabla 1. Departamentos y niveles de complejidad de una muestra de 48 hospitales del Programa de re-estructuración de redes de servicios de Colombia

\begin{tabular}{|c|c|c|c|c|c|c|c|c|}
\hline \multirow{3}{*}{ Departamento } & \multicolumn{6}{|c|}{ Nivel de complejidad } & \multirow{2}{*}{\multicolumn{2}{|c|}{ Total }} \\
\hline & \multicolumn{2}{|c|}{ I } & \multicolumn{2}{|c|}{ II } & \multicolumn{2}{|c|}{ II } & & \\
\hline & $\mathrm{n}$ & $\%$ & $\mathrm{n}$ & $\%$ & $\mathrm{n}$ & $\%$ & $\mathrm{n}$ & $\%$ \\
\hline Antioquia & 3 & 6 & 2 & 4 & 0 & 0 & 5 & 10 \\
\hline Atlántico & 2 & 4 & 1 & 2 & 0 & 0 & 3 & 6 \\
\hline Bogotá & 2 & 4 & 1 & 2 & 0 & 0 & 3 & 6 \\
\hline Boyact & 8 & 17 & 2 & 4 & 0 & 0 & 10 & 21 \\
\hline Caldas & 2 & 4 & 1 & 2 & 0 & 0 & 3 & 6 \\
\hline César & 1 & 2 & 0 & 0 & 0 & 0 & 1 & 2 \\
\hline Choco & 1 & 2 & 0 & 0 & 0 & 0 & 1 & 2 \\
\hline Córdoba & 2 & 4 & 0 & 0 & 0 & 0 & 2 & 4 \\
\hline Cundinamarca & 2 & 4 & 0 & 0 & 1 & 2 & 3 & 6 \\
\hline Huila & 1 & 2 & 0 & 0 & 0 & 0 & 1 & 2 \\
\hline La Guajira & 2 & 4 & 0 & 0 & 0 & 0 & 2 & 4 \\
\hline Magdalena & 3 & 6 & 1 & 2 & 0 & 0 & 4 & 8 \\
\hline N. Santander & 0 & 0 & 0 & 0 & 1 & 2 & 1 & 2 \\
\hline Risaralda & 0 & 0 & 1 & 2 & 1 & 2 & 2 & 4 \\
\hline Santander & 3 & 6 & 1 & 2 & 0 & 0 & 4 & 8 \\
\hline Sucre & 1 & 2 & 0 & 0 & 0 & 0 & 1 & 2 \\
\hline Tolima & 1 & 2 & 1 & 2 & 0 & 0 & 2 & 4 \\
\hline Total & 34 & 71 & 11 & 23 & 3 & 6 & 48 & 100 \\
\hline
\end{tabular}

La prueba piloto del PECASUSS (5) indicó que al menos 70 \% de usuarios estaban satisfechos o muy satisfechos; se calculó que con mínimo 320 usuarios habría un error menor al 5 \%. Para identificar el impacto del Programa en la calidad percibida según la dirección y magnitud de las diferencias significativas entre la línea de base 2006 y las mediciones en 2008 y 2010, en un intervalo del 95 \% de confianza y una potencia de prueba del $90 \%$, el análisis de factores del diseño (objetivos, resultados, recursos) señaló que con 43 usuarios por IPS se tendrían las inferencias requeridas (3).

Para definir la muestra de informantes se usó el Epi-Info. Según el error estándar, la proporción mínima de usuarios satisfechos $(0,7)$ y una confianza 
del 95 \%, se tendrían errores aceptables con 3600 a 4095 encuestados. La representatividad por afiliación al Sistema General de Seguridad Social en Salud-SGSSS, naturaleza del servicio y producción de las IPS se usaron para llegar a usuarios de cualquier edad, sexo, condición social, procedencia y afiliación al SGSSS que en agosto de 2006 recibieron servicios electivos (consulta médica general, especializada u hospitalización) o de urgencias en las IPS, que voluntariamente y con información suficiente aceptaron participar al momento de su egreso del hospital (3).

\section{RESULTADOS}

Con un rechazo de seis potenciales informantes, en los primeros 21 días de agosto de 2006 un total de 4021 usuarios fueron entrevistados. Sus características se reseñan en la Tabla 2.

La percepción de calidad de servicios recibidos por los usuarios encuestados, según los reactivos del PECASUSS (5), reveló los siguientes hallazgos. La percepción de la espera para ser atendidos varió de 0 a 360 minutos, con mediana 30 y media de 55,3 (DE: 64,6). Un grupo de 1017 (25,3 \%) usuarios dijo haber esperado menos de 10 minutos; 1306 (32,5 \%) esperó de 11 a 30; $705(17,5 \%)$ de 31 a 60; 533 (13,2\%) de 61 a 120; 288 (7,2\%) de 121 a 180 y, 171 (4,3 \%) esperaron más de 181 minutos, 3 horas. Estos tiempos de espera se percibieron como muy cortos por 354 (8,8 \%) usuarios; como corto por 1 397 (34,7 \%); ni corto ni largo por 949 (23,6\%); largo por 723 (18,0 \%) y, como muy largo, por los 598 (14,9\%) restantes.

En cuanto al trámite efectuado para la atención, 146 (3,6 \%) lo percibieron como muy sencillo; 2381 (59,2 \%) sencillo; 732 (18,2 \%) ni sencillo ni complicado; 563 (14\%) complicado y, 199 (4,9\%) muy complicado. Un subgrupo de 1715 (42,7 \%) de los 4021 participantes en la medición tuvieron que realizar copagos o pagos moderadores para ser atendidos; a 105 (2,6 \%) les pareció muy barato, a 921 (22,9 \%) barato, a 531 (13,2 \%) ni barato ni caro; a 127 (3,2 $\%)$ caro y solo a $31(0,8 \%)$ les pareció muy caro.

Respecto del estado de la planta física 202 (4,9\%) usuarios lo percibieron como muy cuidado; 2386 (59, 4 \%) como cuidado; 917 (22,8 \%) como ni cuidado ni descuidado; para 441 (11\%) estaba descuidado y a 75 (1,9\%) les pareció muy descuidado. La percepción de los elementos de uso común en salas de espera y habitaciones fue percibida por 122 (3,0 \%) usuarios como muy cómodos; 2479 (61,7 \%) dijeron que eran cómodos; 876 (21,8%) ni 
incómodos ni cómodos; 462 (11,5 \%) incómodos y los 82 (2%) restantes los señalaron como muy incómodos. El aseo de las IPS es muy bueno para 347 (8,6 \%) informantes; bueno para 2536 (63,1 \%); ni bueno ni malo para 846 (21 $\%)$; malo para 246 (6,1 \%) y muy malo para $46(1,1 \%)$.

Tabla 2. Características de una muestra de usuarios de IPS públicas de Colombia

\begin{tabular}{|c|c|c|c|}
\hline Aspecto & n & $\%$ & $\begin{array}{c}\% \\
\text { acumulado }\end{array}$ \\
\hline \multicolumn{4}{|l|}{ 1. Respuestas recibidas } \\
\hline Mury inapropiadas & 35 & 0,9 & 0,9 \\
\hline Inapropiadas & 134 & 3,3 & 4,2 \\
\hline Ni inapropiadas ni apropiadas & 526 & 13,1 & 17,3 \\
\hline Apropiadas & 3092 & 76.9 & 94,2 \\
\hline Muy apropiadas & 234 & 5.8 & 100,0 \\
\hline \multicolumn{4}{|l|}{ 2. Trato de los asistenciales } \\
\hline Muy malo & 16 & 0.4 & 0,4 \\
\hline Malo & 82 & 2.0 & 2,4 \\
\hline Ni malo ni bueno & 466 & 11,6 & 14,0 \\
\hline Bueno & 2755 & 68,5 & 82,5 \\
\hline Mury bueno & 702 & 17,5 & 100,0 \\
\hline \multicolumn{4}{|l|}{ 3. Trato de los administrativos } \\
\hline Muy malo & 15 & 0.4 & 0,4 \\
\hline Malo & 95 & 2,4 & 2,8 \\
\hline Ni malo ni bueno & 620 & 15,4 & 18,2 \\
\hline Bueno & 2934 & 73,0 & 91,2 \\
\hline Muy bueno & 357 & 8.9 & 100,0 \\
\hline \multicolumn{4}{|l|}{ 4. Cooperación entre funcionarios } \\
\hline Muy mala & 17 & 0,4 & 0,4 \\
\hline Mala & 78 & 1,9 & 2,4 \\
\hline Ni mala ni buena & 640 & 15.9 & 18.3 \\
\hline Buena & 3069 & 76.3 & 94,6 \\
\hline Muy buena & 217 & 5,4 & 100,0 \\
\hline \multicolumn{4}{|l|}{ 5. Capacidad de los profesionales } \\
\hline Mury baja & 32 & 0,8 & 0,8 \\
\hline Вaja & 133 & 3,3 & 4,1 \\
\hline Ni baja ni alta & 721 & 17,9 & 22,0 \\
\hline Alta & 2815 & 70,0 & 92,0 \\
\hline Muy alta & 320 & 8.0 & 100,0 \\
\hline \multicolumn{4}{|l|}{ 6. Solución a la necesidad } \\
\hline Muy mala & 38 & 0,9 & 0,9 \\
\hline Mala & 113 & 2,8 & 3,8 \\
\hline Ni mala ni buena & 619 & 15.4 & 19,2 \\
\hline Buena & 2928 & 72,8 & 92,0 \\
\hline Muy buena & 323 & 8,0 & 100,0 \\
\hline \multicolumn{4}{|l|}{ 7. Orientación de cuidadoen casa } \\
\hline Mury insuficiente & 38 & 0,9 & 0,9 \\
\hline Insuficiente & 180 & 4,5 & 5,4 \\
\hline Ni insuficiente ni suficiente & 506 & 12,6 & 18,0 \\
\hline Suficiente & 3047 & 75.8 & 93,8 \\
\hline Mury suficiente & 250 & 6,2 & 100,0 \\
\hline
\end{tabular}


Frente a la atención recibida, 290 (7,2 \%) usuarios dijeron estar muy satisfechos; 2880 (71,6 \%) satisfechos; 649 (16,1\%) ni satisfechos ni insatisfechos; 133 (3,4 \%) insatisfechos y los 69 (1,7 \%) restantes dijeron estar muy insatisfechos. Los usuarios calificaron la calidad del servicio recibido, en una escala de 0,0 a 5,0, con un promedio de 3,98 (DE 0,8). Un grupo de 1014 (25,2 \%) asignaron cinco; $1978(49,3)$ dieron puntajes de cuatro a cuatro nueve; 846 (21\%) de tres a tres nueve; 128 (3,2 \%) de dos a dos nueve; 42 (1\%) de uno a uno nueve y los $13(0,3 \%)$ restantes calificaron este aspecto con valores de cero a cero nueve. La siguiente Tabla resume la distribución de los hallazgos de calidad percibida para las demás dimensiones que son exploradas por reactivos del PECASUSS.

Un subgrupo mayoritario de 3904 (97\%) de los 4021 participantes expresó su motivación, su adherencia para volver a demandar servicios en la IPS en que fueron atendidos; los 117 (3 \%) restantes expresaron no tener la más mínima intención de volver. Entre los usuarios que afirmaron motivación para volver, 1937 (48 \%), señalaron la buena atención, diferentes expresiones del buen servicio, como la razón de esta. Otros 885 (22 \%) refirieron motivación a volver por la necesidad de atender su situación personal de salud o de enfermedad mediante consulta general, de urgencias o controles y tratamiento de una patología o situación crónica. Para otros 669 (17 \%) usuarios la razón de la adherencia a la IPS es la inexistencia de otra opción para atención en salud, bien porque es la única IPS pública en la municipalidad o la única a la que se tiene acceso geográfico, económico o administrativo. En porcentajes menores y decrecientes otros refieren como razón de adherencia la evidente mejora del servicio de la institución; la tradición familiar de demandar atención en esa institución o considerar que es la IPS de personas pobres o en condición asumida de marginalidad social.

Entre los 117 (3 \%) usuarios que expresaron su decisión de no volver a la IPS se identificaron las siguientes razones: en 47 (1,2\%) la mala calidad de la atención por médicos y personal administrativo negligente y deshumano; para $25(0,6 \%)$ la demora en la atención, particularmente en urgencias; en otros 12 $(0,3 \%)$ la prestación de servicios incompletos e, incluso, se registro la negación a volver a esa IPS de un usuario por la muerte de familiar ocurrida, según el, por negligencia en el servicio de urgencias. 
Tabla 3. Aspectos de calidad percibida por usuarios de hospitales de Colombia 2006

\begin{tabular}{|c|c|c|c|}
\hline Variables & $n$ & $\%$ & $\%$ acumulado \\
\hline \multicolumn{4}{|l|}{ 1. Sexo } \\
\hline Masculino & 1205 & 30,0 & 30,0 \\
\hline Femenino & 2816 & 70,0 & 100,0 \\
\hline \multicolumn{4}{|l|}{ 2. Edad Media 37,9 (DE: 15,3 ) } \\
\hline 19 y menos & 365 & 9,1 & 9.1 \\
\hline $20-29$ & 1087 & 27,0 & 36,1 \\
\hline $30-39$ & 918 & 22,8 & 58,9 \\
\hline $40-49$ & 763 & 19,0 & 77,9 \\
\hline $50-59$ & 456 & 11,3 & 89,3 \\
\hline 60 y más & 432 & 10,7 & 100,0 \\
\hline \multicolumn{4}{|l|}{ 3. Servicio } \\
\hline Medicina general & 2137 & 53,1 & 53,1 \\
\hline Medicina especializada & 545 & 13,6 & 66,7 \\
\hline Hospitalización & 226 & 5,6 & 72,3 \\
\hline Urgencias & 1113 & 27,7 & 100,0 \\
\hline \multicolumn{4}{|l|}{ 4. Afiliación al SGSSS } \\
\hline Régimen contributivo & 272 & 6,8 & 6,8 \\
\hline Régimen subsidiado & 2427 & 60,3 & 67,1 \\
\hline Sin afiliación & 1322 & 32,9 & 100,0 \\
\hline \multicolumn{4}{|l|}{ 5. Educación } \\
\hline Ninguna o primaria incompleta & 878 & 21,8 & 21,8 \\
\hline Primaria completa & 2007 & 49,9 & 71,7 \\
\hline Secundaria completa & 999 & 24,8 & 96,5 \\
\hline Técnico-tecnológica & 106 & 2,6 & 99,1 \\
\hline Profesional-postgrado & 31 & 0,9 & 100,0 \\
\hline \multicolumn{4}{|l|}{ 6. Ocupación } \\
\hline Estudiante & 240 & 6,0 & 6,0 \\
\hline Trabajador dependiente & 529 & 13,2 & 19,2 \\
\hline Trabajador independiente & 601 & 14,9 & 34,1 \\
\hline Desempleado & 1034 & 25,7 & 59,8 \\
\hline Pensionado & 14 & 0,3 & 60,1 \\
\hline Otra, mayoritaria amas de casa & 1603 & 39,9 & 100,0 \\
\hline \multicolumn{4}{|l|}{ 7. Estrato } \\
\hline Uno & 2221 & 55,2 & 55,2 \\
\hline Dos & 1499 & 37,3 & 92,5 \\
\hline Tres & 239 & 6,0 & 98,5 \\
\hline Cuatro & 15 & 0,4 & 98,9 \\
\hline Cinco & 3 & 0,1 & 99,0 \\
\hline Seis & 0 & 0,0 & 99,0 \\
\hline Sin dato & 44 & 1,0 & 100,0 \\
\hline
\end{tabular}

De los 4021 encuestados, 2875 (72\%) expresaron opiniones sobre elementos mejorables en las IPS. La mayoría de estos, 25 \%, sugirió agilizar los servicios mediante reducción de: tramites administrativos, exigencia de documentos, del tiempo para efectuar pagos, tener alta o el transcurrido entre la solicitud de una cita médica y la atención efectiva. En segunda instancia los usuarios concentraron sus sugerencias en cuatro campos: primero, la presencia de más personal asistencial en las IPS, en especial médicos generales y especialistas (10\%); segundo, el aumento de las dimensiones físicas y el 
enlucimiento de la infraestructura (7\%); tercero, la mayor dotación en general, en la disponibilidad de ambulancias, de equipos técnicos para la realización de procedimientos diagnósticos o terapéuticos y de mueblería básica para comodidad de los usuarios en términos de silletería, camas, camillas, entre otros (4\%) y, cuarto, la humanización del trato al usuario por personal administrativo o asistencial (3,7 \%).

Otras sugerencias dadas por los encuestados con menor frecuencia fueron: mejorar la iluminación y el aseo general de las instalaciones, en particular de utensilios, cobijas, toallas, sabanas, baños, áreas de ingreso y circulación, tanto en urgencias como en consulta general; agilizar la entrega de resultados y medicamentos; en el caso de IPS con actividades de hospitales universitarios, se registraron pedidos explícitos de usuarios para reducir la presencia de estudiantes y practicantes, pues consideran que afectan la calidad del servicio.

\section{DISCUSION}

Las estimaciones de la línea base 2006, de la calidad percibida por los usuarios de hospitales del Programa, tienen la limitación de ser una aproximación a los valores reales de dicha percepción. Esa limitación fue considerada para ajustar definiciones y conceptos, adecuar el modelo de evaluación (6) y diseñar el muestreo para así garantizar la misma probabilidad de selección a cada institución del marco de elegibles y a cada usuario atendido durante la recolección de datos (3). Las fuentes de error previstas en esta medición fueron potenciales errores del muestreo y errores no muestrales; entre los del muestreo están los del ejercicio probabilístico de entrevistar a una muestra y no al universo de usuarios. Frente a los no muestrales se controlaron los potenciales efectos que en la medición se generan en los procesos para recoger, procesar e interpretar los datos (3).

Una línea de base es indispensable al evaluar la adecuación de procesos y e interpretar la plausibilidad de las evidencias del impacto de iniciativas sociales, programas de salud y salud pública (6). En América, desde 1995, la Organización Panamericana de la Salud-OPS recoge datos de la situación de salud de cada país de la región en el propósito de actualizar el perfil de salud y comparar el avance en cada Estado respecto de otros (7); la OPS también produjo y diseminó en América material para generar líneas de base, aplicable a países con procesos de reforma sectorial (8). En Colombia hay ejercicios de evaluación inicial para medir el desempeño e impacto de intervenciones sociales o sectoriales en salud (9-13). 
En el ámbito de los servicios de salud en Colombia, varias líneas de base y evaluaciones de impacto de programas e intervenciones con referencia a la calidad han sido socializadas. Según el material consultado, los referenciales metodológicos de estos ejercicios han tenido limitados procesos de diseminación y, menos aún, de discusión académica, en particular del desarrollo, adaptación y validación de instrumentos para medir calidad de servicios percibida por los usuarios (14-18). El Departamento Nacional de Planeación-DNP realizó a finales de los 90 del siglo pasado un análisis de las encuestas de calidad de vida para evaluar el impacto del SGSSS en Colombia (14). Con un alcance apenas institucional, Briceño divulgó un modelo para evaluar la calidad de los servicios de salud en una IPS de alta complejidad, en "satisfacción del usuario" según una muestra de 306 usuarios (15). Ambos trabajos tuvieron poca utilidad al diseñar la metodología y discutir la línea de base aquí reportada, por el propósito que tenían, como por la falta o la insuficiente comunicación del proceso de construcción y validación de los instrumentos utilizados.

La Defensoría del Pueblo de Colombia realizó en abril del 2000 la denominada Primera Encuesta Nacional de Calidad en Salud Percibida por UsuariosENCAL I (16). En la perspectiva de la aproximación a la percepción de usuarios planteada en la medición de línea base 2006, objeto del presente artículo y en otro ya comunicado (5), la ENCAL I está inadecuadamente denominada por presentar una incoherencia entre la aproximación "teórica” y los referentes sobre motivaciones, percepciones, conocimientos, y prácticas consideradas en sus objetivos; además, su proceso e instrumental -no validadoindagó, más que la percepción específica de calidad de servicios, un conjunto de dimensiones referidas a informaciones, conocimientos y prácticas, la mayoría en perspectiva de las expresiones de actores de las instituciones y agentes de administradoras del régimen contributivo o subsidiado sobre indicadores de calidad técnica. De cualquier modo la ENCAL I ha sido -hasta ahora- el referente de las mediciones de calidad percibida de servicios de salud para el país (16).

Según la ENCAL I, la percepción de los costos de la atención médica, en particular entre ciudadanos pobres -58\% del total de sus entrevistados- disminuyeron notoriamente por efecto del SGSSS. Aunque con fines de medición diferente, esto se correspondería en parte con la percepción reportada por el subgrupo de participantes de la línea base 2006 que hicieron pagos para ser atendidos, más favorecidos económicamente que quienes en la misma muestra de la línea base no tuvieron que pagar nada, pues en su gran mayoría los pagos efectuados les parecieron baratos. 
La calificación promedio a los servicios recibidos por los encuestados de la ENCAL del 2000 fue de 3,9 un valor que, sin desviación estándar reportada, es apenas 0,8 décimas menor que el promedio de calificación de la línea base 2006, de 3,98 (DE: 0,8). En la ENCAL obtuvieron mejor calificación la atención de médicos generales o especialistas (4,3 para ambos grupos) y peor los servicios de promoción-prevención $(3,0)$ y farmacia $(3,6)$; con una pequeña diferencia, la menor calificación fue dada por usuarios del régimen contributivo cuyas expectativas de calidad podrían ser superiores a las de usuarios del régimen subsidiado o de población vinculada al SGSSS (16).

También en el 2000 se reportaron resultados de la evaluación de la operación del sistema de garantía de calidad para IPS de Colombia (17). Este proyecto desarrolló instrumentos para medir el desempeño de los actores en el marco de incentivos y estrategias que mejoran la calidad de la atención en el SGSSS. Los usuarios en general y usuarias de atención al parto tenían expectativas por servicios con un alto nivel de calidad, generada por relaciones interpersonales "de buen trato y atención del médico, junto con la buena atención, el buen servicio, la amabilidad y la calidad de las enfermeras"; de igual modo se expresó aprecio a la rapidez de las citas y la atención inmediata. Entre las razones de desagrado predominaron las dificultades para acceder a los servicios del sistema, las dificultades con el suministro de medicinas y el disgusto con el costo de los servicios. Es de anotar que las personas de estratos bajos, variable asociada a menor nivel educativo, son sensibles a la facilidad de los trámites, lo cual probablemente reflejaba mayor dificultad de estas personas para "navegar” el SGSSS (17).

A comienzos del 2001 se divulgó la segunda medición del proyecto Municipios Centinela-MC del Programa Sistemas Municipales de Salud-PSMS del entonces Ministerio de Salud de Colombia. Esta medición evaluó resultados y avances en los procesos de la reforma del SGSSS hasta 1998 en 20 municipios en 12 departamentos, que representaban socio demográfica y económicamente el 85\% de municipios del país. La metodología comprendió dos partes, una de seguimiento de procesos; la otra de resultados y efectos de la reforma, intentando conocer el mejoramiento de los sistemas y servicios de salud en términos de equidad, eficiencia y calidad (18).

En calidad se midieron, por un lado, cambios presentados en algunos indicadores de calidad técnica de las IPS y, por el otro, la satisfacción mediante encuestas. Es pertinente señalar a este respecto que el reporte menciona únicamente el rediseño y simplificación de los instrumentos utilizados para la pri- 
mera recolección, sin referirse a un proceso de validación. La percepción de la calidad de servicios fue mayoritariamente calificada como buena sin obtenerse una calificación numérica; el mayor porcentaje de población satisfecha era subsidiada (18). Respecto de esta última experiencia relatada y las otras experiencias previamente discutidas se insiste en la limitada discriminación y alcance interpretativo de una calificación como la reportada de tres opciones de respuesta para valorar la calidad percibida como buena, regular o mala. Y, segundo, en el uso indiferenciado que se hizo en este reporte, así como es frecuente en los otros, de los vocablos satisfacción -con los servicios recibidos- y, percepción -de la calidad de los mismos- como si fuesen sinónimos o equivalentes

Agradecimientos. La información reseñada se tomó, con autorización, del informe de la consultoría 2060685 Fondo Financiero de Proyectos de Desarrollo-FONADE y Universidad de Antioquia, en el marco de ejecución del Convenio de Gerencia Integral del Proyecto 194045 FONADE-Ministerio de Protección Social, Contrato Préstamo 1525/ OC-CO entre la República de Colombia y el Banco Interamericano de Desarrollo-BID.

\section{REFERENCIAS}

1. República de Colombia-Banco Interamericano de Desarrollo. Programa de reorganización, rediseño y modernización de redes prestadoras de servicios de salud. Bogotá D.C.: República de Colombia-BID, Contrato Préstamo BID 1525/OC-CO; 2004.

2. Ministerio de la Protección Social de la República de Colombia. Política Nacional de Prestación de Servicios de Salud. Bogotá D.C.: El Ministerio; 2005.

3. Ministerio de la Protección Social de la República de Colombia. Percepción de la calidad según usuarios de IPS objeto del Programa de reorganización, rediseño y modernización de redes prestadoras de servicios de salud: línea de base 2006 para evaluar el impacto del Programa. Bogotá: Facultad Nacional de Salud Publica "Héctor Abad Gómez" Universidad de Antioquia y Ministerio de la Protección Social; 2006.

4. Sánchez R, Echeverri J. Validación de escalas de medición en salud. Rev Salud Pública (Bogotá) 2004; 6(3):302-18.

5. Cabrera G, Londoño J, Bello L. Validación de un instrumento para medir calidad percibida en hospitales de Colombia. Rev Salud Pública (Bogotá) 2008; 10(3): 443-451.

6. Habicht J, Victora C, Vaughan J. Evaluation designs for adequacy, plausibility and probability of public health programme performance and impact. Int J Epidemiol 1999; 28: 10-8.

7. Organización Panamericana de la Salud. Situación de Salud en las Américas: indicadores básicos. Washington DC: Oficina del director adjunto OPS/OMS; 2005.

8. Organización Panamericana de la Salud. Línea basal para el seguimiento y evaluación de la reforma sectorial. Washington. D.C.: OPS/OMS; 1998. Serie reforma del sector salud.

9. Aldana E, Durán F, Becerra C, Varela J, Vélez E. Línea de base de la encuesta sobre lactancia materna 1978. Bogotá: Instituto SER de investigación; 1979.

10. Gómez LF, Gómez LC, Mateus J, Samper B, Cabrera G. Línea de base para la evaluar impacto de acciones de prevención de factores de riesgo cardiovasculares en la localidad de Santa fe del Distrito Capital. Bogota: Secretaria Distrital de Salud-Fundación FES Social; 2002. 
604 REVISTADE SALUD PÚBLICA · Volumen 10 (4), Septiembre 2008

11. República de Colombia. Programa familias en acción: Línea de base. En: SINERGIA-Sistema Nacional de Evaluación de Resultados de la Gestión Pública. Bogotá: Departamento Nacional de Planeación; 2002.

12. Niño B, Bello M, Londoño S, Docal M, Torres V, Baquero M. Del socavón a la vida: Línea de base para el acercamiento a la erradicación y prevención del trabajo infantil en la minería artesanal colombiana. Bogotá: Proyecto PEPTIMA-Programa PRIAC; 2003.

13. Muñoz E, Gutiérrez $M$, Arango $O$, Guerrero R. Conocimientos, actitudes y prácticas sobre violencia en Bogotá, Cali y Medellín: Línea de base. Bogotá: Ministerio de la Protección Social-Instituto CISALVA Universidad del Valle; 2004.

14. Departamento Nacional de Planeación. Análisis de las encuestas de calidad de vida para evaluar el impacto del nuevo Sistema General de Seguridad Social en Salud en Colombia 1993-1997. Bogotá; 1998.

15. Briceño C. Modelo para evaluar calidad del servicio en una IPS de alta complejidad, en su componente de "satisfacción del usuario". Rev Col Marketing 2001; 2(3): 1-9.

16. Defensoría del Pueblo. Primera Encuesta Nacional de Calidad Percibida por los Usuarios de Servicios de Salud. Bogotá; 2000.

17. Ministerio de Salud. Programa de Apoyo a la Reforma en Salud-PARS. Evaluación y ajuste de procesos, estrategias y organismos encargados de operación del sistema de garantía de calidad para IPS's. Bogotá: Centro de Gestión Hospitalaria-Canadian Council on Health Services Acreditation-Qualimed; 2000.

18. Ministerio de Salud. Programa Sistemas Municipales de Salud-PSMS. Proyecto Municipios Centinela, segunda medición evaluativo de los resultados y avances en los procesos con la implantación de la reforma del Sistema General de Seguridad Social en Salud en 20 municipios del país, 1995-1998. Bogotá; Diciembre de 2000. 